\title{
Criminologie
}

\section{Stratégies de changement et politiques pénales}

\section{André Normandeau}

Volume 19, numéro 1, 1986

Politiques et pratiques pénales. 25 ans de réflexion et d'action

URI : https://id.erudit.org/iderudit/017231ar

DOI : https://doi.org/10.7202/017231ar

Aller au sommaire du numéro

Éditeur(s)

Les Presses de l'Université de Montréal

ISSN

0316-0041 (imprimé)

1492-1367 (numérique)

Découvrir la revue

Citer cet article

Normandeau, A. (1986). Stratégies de changement et politiques pénales. Criminologie, 19(1), 171-187. https://doi.org/10.7202/017231ar

\section{Résumé de l'article}

This article examines criminal justice policies and strategies of change, from agenda setting to decision-making, implementation and evaluation. Since 1960, change has been incremental, almost without regard to the predominant liberal or conservative ideological strategies. The author maintains, even if it is an old cliché, that reforming society is a better social policy than reforming the criminal justice system... if we have a choice! 
André Normandeau*

This article examines criminal justice policies and strategies of change, from agenda setting to decisionmaking, implementation and evaluation. Since 1960, change has been incremental, almost without regard to the predominant liberal or conservative ideological strategies. The author maintains, even if it is an old cliché, that reforming society is a better social policy than reforming the criminal justice system... if we have a choice!

\section{INTRODUCTION}

Au moment où la criminologie québécoise, du moins au niveau de son expression universitaire en enseignement et en recherche, fête ses vingt-cinq (25) ans de productivité officielle (1960-1985), il nous apparaît pertinent de tracer un bilan critique des politiques pénales, particulièrement en matière correctionnelle adulte, au Québec et au Canada depuis 1960. En effet, l'hypothèse veut qu'un certain lien existe entre les travaux scientifiques, d'une part, et les politiques, d'autre part, comme si l'évolution des «idées» et des «faits» relevait d'une dialectique rigoureuse conduite par un fil d'Ariane symbolique, selon l'image de la mythologie grecque où Ariane donna à Thésée le fil qui lui permit de sortir du labyrinthe, après avoir vaincu le minotaure. Ce fil, nous nous en doutons bien, est plutôt mince. D'un côtć, les universitaires et les chercheurs en criminologie ont souvent l'impression que leurs idées et les résultats de leurs travaux ne sont pas utilisés par les administrateurs publics et les praticiens qui travaillent dans le domaine de la justice pénale: de l'autre, ces derniers ne manifestent pas d'intérêt particulier pour la théorie et la recherche et préfèrent souvent se fier au sens commun et à l'expérience du vécu. Nous nous retrouvons ainsi entre deux solitudes.

Pourtant, la réalité est plus complexe, particulièrement au royaume de la criminologie et de la justice. En effet, la crimino-

- André Normandeau, directeur, Centre international de criminologic comparée, Université de Montréal. 
logie est à la fois une science et un art, une discipline fondamentale et appliquée, au carrefour du droit, de la sociologie et de la psychologie. A ce titre, la criminologie québécoise s'est distinguée par une volonté d'allier la théorie à la pratique, "pour le meilleur et pour le pire». Nous renvoyons le lecteur à l'histoire de cette criminologie qui est en quelque sorte une toile de fond où se dessine, au moins en partie, le portrait des politiques québécoises et oanadiennes (Criminologie, 1977; Szajo, 1978; Bertrand et Normandeau, 1984; LeBlanc et Szabo, 1985).

Par ailleurs, le bilan «descriptif» des politiques a déjà été tracé autant pour le Québec (Prévost, 1970; Gauthier, 1985) que pour le Canada (Ouimet, 1969; Brodeur et Landreville, 1979; Griffiths, Klein et Verdun-Jones, 1980; Woods et Sim, 1981; Ekstedt et Griffiths, 1984). Le lectcur est invité à s'y référer. Pour notre part. nous partons de cette matière première pour esquisser le portrait idćologique de «certains» changements en matière de politiques pénales. Trois perspectives s'offrent à notre regard à ce sujet : a) la perspective conservatrice; b) la perspective libérale-pluraliste; et c) la perspective radicale-élitiste (Shover, 1979). La perspective radicale est importante (Gosselin, 1977) et mériterait un long exposć que nous réservons pour une autre occasion. Compte tenu que les perspectives conservatrices et libérales sont prédominantes pour l'ensemble de l'Amérique du Nord, autant au Québec qu'au Canada et aux États-Unis, nous les utiliserons comme toile de fond à notre analyse.

Auparavant, toutefois. il est nécessaire, à notre avis, de résumer le processus politique qui gère l'élaboration des politiques pénales en Amérique. comme d'ailleurs dans la plupart des pays démocratiques, afin de comprendre un tant soit peu la dynamique du changement. à cheval, selon l'expression de Szabo (1969), entre la "néoténie» (une orientation-favorable au changement) et le "misonéisme» (la résistance au changement).

\section{L'ÉLABORATION DES POLITIQUES : LE MODĖLE DE L'ÉTAPISME}

Nous avons eu trop tendance en criminologie à étudier les politiques pénales en «seire-chaude». Comme les politiques pénales s'insèrent à l'intérieur du cadre plus vaste des politiques sociales et que ces dernières s'insèrent elles-mêmes au sein du cadre global des politiques publiques. le modèle d'analyse qui nous intéresse 
est également celui que les politologues appliquent à l'ensemble de l'administration publique.

Quelles sont les origines des politiques qui guident les gouvernements? Pourquoi les gouvernements poursuivent-ils ces politiques? Quelles sont les conséquences de ces politiques? Comment les politiques sont-elles évaluées, modifićes, abandonnćes?

Pourquoi étudier les politiques? Traditionnellement, les citoyens font l'hypothèse que lorsqu'une loi est votée (ou un règlement édicté ou une directive élaborée) et que l'argent pour sa mise en vigueur est alloué, il s'ensuit automatiquement que les objectifs recherchés sont atteints. Les citoyens des pays démocratiques sont optimistes, règle générale. On pense que la pauvreté peut être éliminée, le chômage réduit de façon significative, la criminalité sensiblement diminuée... Depuis quelques années, toutefois, les responsables des politiques sont mis au pilori. On exige d'eux, de plus en plus, de rendre des comptes sur l'efficacité et l'efficience des services publics. Faute de quoi la révolte des contribuables s'exprime par des demandes de coupures budgétaires gouvernementales, illustrée par la "proposition 13» en Californie au tournant des années 80 .

L'étude des politiques est donc pertinente et utile non seulement pour des raisons scientifiques mais également pour des raisons professionnelles. On exigera à l'avenir de la part des administrateurs publics et des praticiens des justifications de leurs missions et de leurs actions.

Cependant, l'analyse des politiques n'est pas une panacée et il n'y aura pas de miracle. Certains problèmes de justice, par exemple, ne peuvent être réglés, compte tenu des conflits de valeurs inhérents aux sociétés démocratiques ainsi que du dilemme ou de l'équilibre entre les libertés individuelles et les libertés collectives. De plus, il y a des limites attachées aux méthodes de recherche en sciences humaines et sociales. Le criminologue ne doit pas être l'apprenti-sorcier qui sert de «caution» scientifique aux administrateurs et aux politiciens qui justifient quelquefois leurs décisions sur la foi de données empiriques incomplètes qu'ils ont sélectionnées eux-mêmes. Mais si les données sont utilisées avec circonspection, elles peuvent jeter un éclairage pertinent et permettre aux décideurs de remplacer le "pile ou face» inacceptable sur lequel ils basent souvent les orientations et les solutions qu'ils retiennent. 
L'analyse du processus politique a été maintes fois décrite (American Behavioral Scientist, 1983; Anderson, 1980; Riverin, 1981: Rocher, 1981) et adaptée pour le champ de la justice pénale (Feeley et Sarat, 1980; Solomon, 1981, 1983; Szabo, 1981; Waller, 1979, 1982).

Qu'il nous suffise de rappeler au tableau $A$ que ce processus est découpé en cinq (5) phases successives : 1) la formation d'une politique; 2) sa formulation; 3) la prise de décision; 4) sa mise en vigucur; 5) son évaluation et une certaine reformulation. Les politologues américains regroupent à l'occasion la formation et la formulation d'une politique sous l'étiquette : "agenda setting»; la prise de décision est étudiée à titre de "decision-making»; et la mise en vigueur ou mise en œuvre sous l'appellation: «implementation». L'évaluation (et la reformulation) en est évidemment la phase terminale. Un tel processus relève d'un modèle idéal à la Weber. Ce modèle rationnel n'est pas appliqué dans la réalité et il s'oppose en fait au modèle couramment utilisé en pratique, celui de l'étapisme ou du gradualisme (incremental). Lindblom (1980) en a résumé les grandes lignes au tableau B (Riverin, 1981, p. 50) .

\section{Tableau $A$}

Phases du processus politique (politiques pénales...)

1. Formation d'une politique

- Idées initiales de changement (individus et groupes)

- Désirs préliminaires exprimés par les groupes de pression

- engagements électoraux d'un parti politique

- étude de la question : problématique

2. Formulation d'une politique

- Groupe de travail; commission d'enquête

- Livre vert et livre blanc

- Avant-projet et projet de loi; commission parlementaire

- Étude des options et des alternatives

3. Mise au point d'une politique : la prise de décision

- Conséquences des options et des alternatives sur les plans suivants :

- démocratie et libertés individuelles et collectives

- administration publique et personnel disponible

- finances publiques

- Choix et prise de décision 
4. Élaboration, mise en vigueur ou en cuvre et exécution du plan d'action

- Programmation et implantation par étapes

- Budgétisation

- Le contexte organisationnel et le contexte politique

— Les fonctions et les qualités de l'administrateur public

5. Évaluation des résultats

- Conformité à la législation et à la réglementation

- Vérification financière

- Analyse de la mise en œuvre des interventions

- Analyse de l'impact et reformulation de la politique

\section{Tableau $B$}

Comparaison des méthodes rationnelle et gradualiste

1. La méthode rationnelle globale

- La clarification des valeurs ou des objectifs recherchés constitue un préalable à l'analyse empirique de diverses solutions ou politiques

- Le processus décisionnel chemine dans un continuum et une analyse des fins et des moyens. On isole d'abord les fins poursuivies et on recherche ensuite les moyens permettant de les atteindre.

- La valeur d'une politique est plus ou moins élevée selon que cette politiques s'avère ou non le moyen le plus approprié d'atteindre les objectifs.

- L'analyse est globale. Elle prend en compte chaque facteur pertinent.

- Cette approche se fonde fortement sur la théorie.

2. La méthode gradualiste (ou des comparaisons successives)

- Le choix des objectifs et l'analyse empirique de l'action nécessaire ne se distinguent pas vraiment l'un de l'autre, ils s'enchevêtrent constamment.

- Puisque les fins et les moyens ne sont pas distincts, il est souvent inadéquat ou très partiellement utile de les analyser.

- Une politique est bonne dans la mesure où les analystes s'accordent sur son contenu, cet accord ne préjugeant pas que ces mêmes analystes estiment que c'est le moyen le plus approprié pour l'atteinte d'un objectif. 
- L'analyse est limitée sur le plan de la rigueur :

- on néglige certaines conséquences majeures possibles

- on néglige d'envisager d'autres politiques possibles

- on ne se préoccupe pas de certaines valeurs, ni de certaines quantités qui pourraient être affectées.

- Des comparaisons successives réduisent fortement ou éliminent le recours à la théorie.

Lorsque nous analysons les politiques pénales au Québec et au Canada depuis 1960, il n'est pas difficile d'y reconnaître le modèle de l'étapisme. Comme il est difficile d'arriver à un consensus sur les valeurs et les objectifs à poursuivre, les politiciens et les administrateurs choisissent souvent des politiques sans en avoir au préalable clarifié les objectifs. La discrétion des gestionnaires concernant leurs objectifs s'explique en partie par le désir d'éviter une trop grande polarisation organisationnelle, le désir d'éviter d'offrir des points d'appui à l'opposition et le désir d'éviter une trop grande rigidité de fonctionnement.

Les limites intellectuelles de l'humain et l'insuffisance de l'information rendent impossible l'utilisation de la méthode rationnelle globale pour l'analyse de problèmes complexes, de sorte que face à de tels problèmes l'administration doit trouver des moyens de simplification. Il semble que ce soit par la méthode gradualiste que l'administrateur arrive à faire cette simplification. Cette approche se limite en effet à considérer des politiques qui diffèrent peu de celles qui existent. Cette limitation réduit le nombre de choix à analyser et simplifie ainsi l'analyse. Celle-ci se limite aux aspects par lesquels le choix proposé et ses conséquences diffèrent du statu quo.

D'après lindblom (1980), dans les régimes démocratiques les politiques se modifient presque exclusivement par le biais d'ajustements à la marge. De cette manière, on minimise les risques en se limitant à n'apporter que de petits changements successifs atux politiques qu'on veut modificr.

Ce processus décisionnel en est donc un de rapprochements successifs vers des objectifs qui changent eux-mêmes constamment. L'administrateur qui a expérimenté cette réalité s'attend donc que ses décisions n'alteignent qu'une partie des objectifs qu'il s'est proposés, mais il s'attend aussi que des conséquences qu'il aurait souhaité éviter se produisent. Cela confirme que de procéder par 
le moyen de changements à la marge évite les erreurs coûteuses et trop durables.

L'administrateur dispose, pour le guider dans son approche étapiste, des expériences précédentes qu'il a vécues. Celle-ci lui indiquent les conséquences probables de certains types de décisions. En outre, il n'est pas nécessaire pour lui de faire des changements draconiens qui requerraient des prévisions beaucoup trop poussées, puisqu'il ne considère pas que sa décision soit une solution finale au problème qui le préoccupe. Il sait que sa décision ne constitue qu'un pas en avant qui peut être rapidement suivi par d'autres. Enfin, il peut remédier aux erreurs passées rapidement au moins beaucoup plus rapidement que si ses décisions consistaient en des changements en profondeur très étalés dans le temps.

Lorsque nous regardons à la lumière de cette approche l'évolution, par exemple, du secteur correctionnel adulte, l'envers du décor est le suivant : toutes les politiques correctionnelles ont tendance à se ressembler; les innovations sont rares; la créativité est mince; les résistances au changement (misonéisme) sont fortes même sous un vernis de changement (néoténie). L'administrateur "correctionnel» (Gauthier, 1986) qui a vécu les changements depuis 1960 est relativement satisfait de l'évolution du Québec à ce chapitre : il parle d'alternatives à l'emprisonnement, de la probation (depuis 1968) et des libérations conditionnelles (depuis 1978), du développement des maisons de transition et de la participation communautaire (depuis 1975)... Or, il en est fier et il a probablement raison aux yeux d'une majorité d'administrateurs et de praticiens du secteur correctionnel adulte, car il s'agit bel et bien de réalisations concrètes conquises «pouce par pouce». Malheureusement, cette "politique des petits pas» n'a pas sensiblement modifié la cheville ouvrière du système pénal québécois puisque, par exemple, les taux d'emprisonnement au Ouébec sont les mêmes aujourd'hui que ceux d'il y a 10 ou 15 ans, c'est-à-dire 100 prisonniers par 100000 habitants. Aurions-nous élargi ainsi. malgré nos bonnes intentions, le filet du contrôle social?

En fait, ce qui est remarquable c'est que les résultats des politiques pénales au Québec et au Canada sont relativement homogènes depuis 1960, quelles que soient les orientations idéologiques, la couleur politique des partis au pouvoir ou les intérêts personnels des décideurs, des administrateurs et des praticiens. Les succès de la réforme pénale ne sont pas très élevés. Certes, il y a 
de temps en temps une mini-réforme ici et là. Est-ce suffisant? Sans compter que le sens de ces réformes risque de s'éloigner d'unc certaine tradition criminologique progressiste au cours des prochaines années avec l'arrivéc au pouvoir, au propre et au figuré, d'une perspective de plus en plus conservatrice en matière de politiques pénales et correctionnclles.

\section{LA THÉOLOGIE DU CONTRÔLE SOCIAL: LES CONSERVATEURS ET LES LIBÉRAUX}

Les sondages d'opinion indiquent toujours que le contrôle de la criminalité est un problème important. Depuis 1960, des changements en profondeur des politiques en matière de contrôle social ont été suggérés, d'abord d'un point de vue libéral et, plus récemment, d'une perspective conservatrice. Nous utilisons évidemment ces expressions dans le sens propre et nord-américain des idéologies libérales et conservatrices et non dans le sens des partis politiques qui, au Québec et au Canada, s'identifient avec ces étiquettes, même s'il y a, à l'occasion, un recoupement entre ces idéologies générales et certains aspects des programmes des partis politiques.

Le problème de la criminalité envahit notre pensée. Nos politiques pénales relèvent de la faillite intellectuelle. Nous n'avons pas de politiques crédibles pour contrôler la criminalité. On nous offre, en place et lieu, une série de recettes de désespoir qui nous promettent des solutions rapides et faciles. La plupart d'entre elles ne fonctionneront jamais et plusieurs même sont franchement dangereuses. Depuis les années 60 , nous avons eu droit à des "guerres» contre la pauvreté, contre le chômage, contre la criminalité. Non seulement ces «guerres» n'ont-elles pas contrôlé un tant soit peu la criminalité mais elles ont créé de faux espoirs. Le résultat net : un public frustré et en colère qui demande, à la Shakespeare, "une livre de chair». Nous avons tous perdu une certaine habileté à envisager de façon rationnelle le contrôle de la criminalité. Évidemment, les réponses ne sont pas toujours faciles. On ne contrôle pas n'importe qui, n'importe quand, n'importe comment. En démocratic, il y a des limites au contrôle de la criminalité. Certaines propositions auraient sûrement un effet sur le niveau de la criminalité, mais nous ne pouvons pas sérieusement les soutenir. Par exemple, nous pourrions exécuter sommairement 
les trafiquants de drogues. Nous pourrions emprisonner tous les jeunes délinquants coupables d'un crime de violence. Des principes de justice, d'équité et de décence élémentaire nous retiennent.

La plupart des propositions avancées par les politiciens et les «experts» en matière de justice "ne marchent pas», pour reprendre le diagnostic célèbre de Martinson (1974). Ils ne réussissent tout simplement pas le test de réduire la criminalité. Ces politiques relèvent de la "pensée magique». Elles sont souvent non pertinentes : on ne peut pas les mettre en vigueur et elles n'auraient aucun effet, de toutes façons, sur le taux de la criminalité. $\grave{A}$ l'occasion, elles pourraient réussir à contrôler la criminalité, mais le prix serait exorbitant sur les plans économique, social, culturel, et même... politique. D'autres propositions offrent des avantages mais n'ont aucun effet sur le contrôle : elles peuvent améliorer, par exemple, la qualité de la justice, sans conséquence, toutefois, sur le contrôle de la criminalité.

Nos connaissances sur la criminalité et la justice ont augmenté considérablement depuis 1960. Mais elles nous ont éclairés surtout par la négative. Nous avons appris ce qui ne fonctionnait pas. En 1970, Norval Morris et Gordon Hawkins publiaient un livre intéressant et engagé : The Honest Politician's Guide to Crime Control. En 1975, ils récidivaient avec: $A$ Letter to the President of the United States About Crime Control. À cette époque, les deux criminologues ne proposaient rien de moins qu'un «médicament contre la criminalité». Non pas une potion subito ou une panacée foudroyante mais plutôt un régime législatif et administratif qui réduirait la criminalité. Aujourd'hui, il est douteux qu'une telle prétention serait réitérée ni par eux ni par aucun autre «expert» sensé et raisonnable. La recherche tout azimut des années 60 et 70 nous a appris, en effet, qu'il faut se méficr des «médicaments anticrime». Malheureusement, cette leçon n'a pas été retenue par tout le monde. L'appétit du public pour les remèdes instantanés à ce sujet est presque insatiable et les charlatans de la justice ont beau jeu d'en profiter pour étaler devant la galeric de l'opinion publique des promesses extravagantes sans lendemain. La partisanerie politique se met aussi de la partie. Le débat politique ne fait que mêler les cartes et les citoyens davantage. Il est triste de constater que ni les conservateurs ni les libćraux n'ont de propositions valables dans leurs carquois politiques. Ils sont souvent coupables de colporter des sottises au sujet du contrôle de la criminalité. 
En ce moment, l'initiative politique est dans le camp des conservateurs. Leurs idées sont à la mode et certaines législations récentes aux États-Unis les prolongent dans le feu de l'action: détention préventive; neutralisation sélective; sentence déterminée; abolition des libérations conditionnelles; le retour de la peine de mort; pouvoirs accrus pour la police et les procureurs de l'État; des droits nouveaux pour les victimes... Cette hégémonie actuelle de la pensée conservatrice, illustrée de façon brillante dans les écrits de James Q. Wilson $(1975,1983)$, par exemple, doit être replacée dans le contexte des hauts et des bas, des flux et des reflux des politiques en matière de justice au vingtième siècle. Comme l'a analysé David Rothman (1980), du moins pour la scène américaine, les réformes libérales ont tenu le haut du pavé du début du siècle à la Première Grande Guerre de 14-18. Une réaction conservatrice de type «law and order» lui a succédé pendant les années 20,30 et 40 . Le libéralisme reprit du poil de la bête pendant les années 50 et domina le champ de la justice pendant les années 60.La grande commission américaine Katzenback (1967) sur la justice en est le reflet le plus fidèle. Au Canada, le Comité Ouimet (1969) et au Québec, la Commission Prévost (1979) s'inscrivent également sous cette philosophie réformistelibérale. Les idées conservatrices revinrent à la mode dans les années 70 et dominent nettement maintenant les années 80 . Les «nouvelles» idées conservatrices nous apparaissent «fraiches et pimpantes", excitantes même, en partie à notre avis parce qu'elles n'ont pas été encore évaluées en profondeur. En comparaison, les idées libérales nous enthousiasment beaucoup moins, car l'évaluation des mesures dites libérales a déjà été faite en bonne partie et les résultats sont décevants. que ce soit : la déjudiciarisation, la probation, la réhabilitation en prison, les libérations conditionnelles, les alternatives communautaires à l'emprisonnement... De méchantes langues pourraient lancer le quolibet suivant: les libéraux n'ont plus d'idćes alors que les conservateurs ont des idées dangereuses!

Évidemment, les catégories «libérales» et «conservatrices» sont un peu grossières. Inćvitablement, des distinctions plus subtiles sont obnubilées. En théorie, les membres de chaque idéclogie partagent un certain nombre de "crovances». Les conservateurs proposent de raffermir la "machine judiciaire». Nous pouvons, disent-ils, réduire la criminalité en attrapant plus de criminels, en les trouvant coupables en plus grand nombre. en les punissant de 
façon plus sévère. Les deux symboles des politiques conservatrices sont l'effet intimidant de la peine et la ncutralisation des criminels. La «machine» est affaiblie par toutes sortes de technicalités légales qui permettent aux criminels de s'en sortir; qu'à cela ne tienne. l'agenda conservateur a l'intention d'y remédier.

Les politiques libérales, à leur tour, s'intéressent à «remodeler» le délinquant et le criminel, à "moderniser» et à "humaniser» le système de justice, et à «changer» un tant soit peu la société. La théorie centrale des politiques libérales, la réhabilitation, embrasse une panoplie de programmes aptes à altérer le comportement des criminels et à les transformer en citoyens responsables. Pendant ce temps, on favorise aussi certaines réformes sociales en pensant éliminer ainsi les racines des injustices à la base de la conduite criminelle. On souhaite élargir le corridor des «opportunités» éducationnelles et économiques afin de permettre aux gens de choisir un mode de vie valorisant et ainsi mieux résister aux tentations de nature criminelle.

En pratique, la polarisation idéologique libérale-conservatrice est difficile quelquefois à discerner. Mêmes les étiquettes politiques annonçent des couleurs mixtes: on parle ainsi au Québec et au Canada du Parti «progressiste»-conservateur! Les différences, même en matière de politiques sociales et économiques, sont minces. Comme le disait l'ex-premier ministre Pierre Trudeau, «on gouverne à l'extrême centre»! Il en est ainsi en matière de justice. Une prison est une prison. Le jargon bureaucratique officiel écrit : «un établissement de détention» ou encore «un centre correctionnel». Mais les juges, le public et les prisonniers ne s'y trompent pas : la prison est un lieu de punition. La prison est-elle alors le reflet d'une politique libérale ou conservatrice?

Ces étiquettes ne collent pas toujours parfaitement à la peat d'un individu, comme le gant de l'histoire. Un individu est complexe. Il a parfois des idées contradictoires, libérales le matin, conservatrices le soir, libérales à vingt-cing ans, conservatrices à cinquante. Aux États-Unis, James Q. Wilson, un conservateur renommé, est un ancien libéral qui a "confessé ses fautes». Il en est ainsi au Québec du criminologue Maurice Cusson (1983) qui est en matière de justice un conservateur affiché, sans peur et sans reproche, après une période libérale classique à l'image de la criminologie québécoise des années 60 et même 70 . En sens contraire, l'un des conservateurs éminents des années 70 , le juge en 
chef de la Cour suprême des États-Unis, Warren Burger, a changé son fusil d'épaule et défend depuis le tournant des années 80 la vieille idée libérale de la réhabilitation par le travail en prison.

Malgré ces ambiguïtés, la classification libérale-conservatrice cst utile en Amérique du Nord pour comprendre les principales politiques pénales véhiculées par les intervenants en matière de justice. Walker (1980, 1985 parle à ce sujet d'une "théologie du contrôle social». Le problème de toutes les politiques de contrôle de la criminalité est celui du triomphe de la foi sur les œuvres, de la théorie sans fondement sur les faits empiriques débusqués par la recherche criminologique.

Pour les libéraux comme pour les conservateurs, certaines idées sont littéralement des articles qui relèvent de la foi, de la religion, de la théologie. Leurs croyances relèvent de la conviction religieuse. Elles ne peuvent être confirmées ni infirmées par une argumentation serrée et une évaluation sérieuse de la réalité. Il serait plus facile de prouver l'existence de Dieu! Près de trois Québécois sur quatre sont aujourd'hui favorables à la peine de mort parce que, pensent-ils, cette peine est intimidante et efficace. Cette croyance persiste contre vents et marées en dépit des données empiriques à l'effet contraire. Les conservateurs appuient donc en majorité la peine capitale. D'autre part, plusieurs libéraux sont encore favorables, sans arrière-pensée, à la réhabilitation, malgré les recherches des années 70 et l'évaluation scientifique négative de la plupart des programmes. Les théologies du contrôle social influencent en profondeur le citoyen moyen parce qu'elles reflètent souvent sous forme d'une "gestalt» idéale les aspirations et les désirs de ses adeptes.

\section{LA THÉORIE ET LA PRATIQUE : \\ DE LA CONSCIENCE ET DE LA COMMODITÉ}

Malgré ces orientations de base différentes, nous constatons, à la ligne d'arrivée, que les réformes conservatrices et libérales se ressemblent souvent comme deux chats siamois. Pourquoi en est-il ainsi? Nous avons déjà énoncé une hypothèse d'explication dans la première partie de cet article en décrivant l'élaboration des politiques et le modèle de l'étapisme. Entre la théorie rationnelle et la pratique gradualiste des compromis, le législateur et l'administrateur choisissent les milliers de petits compromis successifs. La prise de décision en matière de politiques pénales relève donc en 
démocratie de perspectives «centristes». Or, au centre, «tout le monde est beau, tout le monde est gentil», et il s'agit souvent du seul consensus possible, ni-chair ni-poisson, ni vraiment conservateur, ni vraiment libéral. Faut-il pleurer, faut-il en rire ou simplement constater qu'en démocratie de type pluraliste c'est la solution à retenir puisque la politique est l'art du possible?

Une autre hypothèse d'explication de ces ressemblances, celle de David Rothman (1980), est en fait une hypothèse complémentaire à la précédente. Une étude historique de la justice aux ÉtatsUnis l'amène à conclure que chaque génération est réformiste, à sa façon, et qu'il y a simultanément des réformistes-progressistes de type conservateur et de type libéral. De plus, chaque génération suscite des réformateurs-théoriciens et des réformateurs-praticiens. Chaque génération secrète des réformateurs qui attachent beaucoup d'importance aux principes et à leur «conscience» alors que d'autres pratiquent l'art de ce qui est possible de réaliser, de ce qui est commode (convenience) et réaliste, compte tenu des résistances au changement, des budgets disponibles, des orientations favorables au changement mais contradictoires au niveau des principes retenus et des moyens suggérés. D'où le titre de son livre sur la dynamique des deux concepts clés de toute réforme : Conscience and Convenience. Cette "alliance» ne pouvait et ne donne toujours qu'une version hybride des «belles réformes» initialement envisagées.

La question à poser est alors vraiment celle-ci : une réforme pénale basée sur une stratégie de changement où tant de compromis ont lieu est-elle encore vraiment une réforme car, lorsqu'il y a rencontre entre la "conscience» et la "commodité», c'est la commodité qui emporte le gâteau? Une réforme tronquée vaut-elle vraiment mieux que le statu quo? Peut-on imaginer un jour des politiques pénales vraiment différentes des politiques d'hier et d'aujourd'hui? Y a-t-il vraiment des «alternatives» pénales à la Tomasic et Feeley (1982) ou à la Abel (1982)? Ou même des alternatives vraiment non pénales à la Mathiesen (1974), à la Christie (1981), à la Hulsman (1982) ou à la Landreville (1983)? Michel Foucault nous répond déjà que les alternatives, non seulement à l'emprisonnement mais par extension les autres alternatives au sens large également, ne sont qu'une «autre façon de surveiller et punir», David Rothman, de son côté, affirme. malgré sa critique historique dévastatrice, que «le changement est tout de même possible»; difficile, exigeant mais possible. 
Entre la version pessimiste de Foucault et la version optimiste (mais critique et réaliste) de Rothman, le lecteur fera son propre lit et tirera ses propres conclusions.

Le lecteur ne sera pas surpris de savoir que je me range dans l'équipe de Rothman. Au niveau des stratégies de changement et des politiques pénales, je suis un «étapiste» et un "gradualiste». J'ai des principes (conscience) mais je suis aussi un homme d'action, à ma façon, et je m'accommode (convenience) de certains compromis centristes. En retour, je ne suis ni «conservateur» ni «libéral» et je cherche une troisième voie que l'on appelle quelquefois «social-démocrate». I'ai déjà écrit (Normandeau, 1975, 1976. 1977, 1978, 1980; Normandeau et Poirier, 1985) et le pense encore, même si c'est un cliché "gros comme une prison», qu'un vrai changement est avant tout un changement de société et après, un changement du système pénal. Je songe ici à des réformes politiques, économiques, sociales et culturelles "profondes».

Prendre conscience de cela, c'est en même temps prendre conscience qu'il est vain d'essayer, par le biais du système pénal, de corriger les injustices sociales qui auront été suscitées en dehors de lui. De même qu'il ne suffit pas de construire des villes modèles pour rendre leurs habitants heureux, de même ne suffit-il pas de construire une justice modèle pour que la société soit juste. Il y a même, à la limite, quelque chose de mystificateur dans une telle entreprise de réforme en profondeur, si l'on cherche par là à convaincre la population que la rénovation du système de justice constitue le remède à ses maux. Cela ne veut pas dire qu'il faille carrément. et d'entrée. renoncer. Cela veut dire qu'il faut se garder de traiter le système pénal hors de son contexte : le modifier, oui, à condition que l'on modifie aussi ce qui l'entoure, afin de donner tout leur sens et toute leur ampleur aux réformes spécifiques. La justice est une affaire politique et sociale, non judiciaire.

Mais je m'égare. Ce thème devra faire l'objet d'un autre article... peut-ître au cinquantième (50) anniversaire de la criminologie au Qućbec en l'an de grâce 2010! 


\section{REFERENCES}

ABEL, R. (édit.) (1982), The Politics of Informal Iustice, New York. Academic Press.

AMERICAN BEHAVIORAL SCIENTIST (1983), Social Sciences and Policy Making: The Use of Research by Government Commissions, Numéro thématique, 26, 5.

ANDERSON, I. (1980), Public Policy-Making, New York, Holt, Rinehart and Winston.

BERTRAND, M.A. et A. NORMANDEAU (1984), "Les sciences humaines à l'assaut (ou au service?) des appareils de justice pénale au Québec». in G.H. Lévesque et coll., Continuité et rupture : les sciences sociales au Québec, Montréal : P.U.M., p. 319-336.

BRODEUR, J.P. et P. LANDREVILLE (1979), Finalités du système de l'administration de la justice pénale et planification des politiques. Les Cahiers de l'École de criminologie, Université de Montréal.

CHRISTIE, N. (1981), Limits to Pain, New York, Columbia University Press.

CRIMINOLOGIE (1977), la Criminoloxie au Québec. Numéro thématique, 10, 1, Montréal : P.U.M .

CUSSON, M. (1983), le Contrôle social du crime, Paris, Presses universitaires de France.

EKSTEDT, J. et C. GRIFFITHS (1984), Corrections in Canada: Policy and Practice, Toronto, Butterworths.

FEELEY, M. et A. SARAT (1980), The Policy Dilemma: Federal Crime Policy and the Law Enforcement Assistance Administration : 1968.1978. Minneapolis, University of Minnesota Press.

GAUTHIER, M. (1986), "Bilan et évaluation des politiques et des pratiques en matière correctionnelle adulte au Québec : 1960-1985». Criminologie (ce numéro).

GOSSELIN, L. (1977), les Pénitenciers : un système à abattre, Montréal, Les Éditions Saint-Martin.

GOSSELIN, L. (1982), Prisons in Canada, Montréal, Black Rose (trad.).

GRIFFITHS, C., J. KLEIN et S. VERDUN-JONES (1980), Criminal Justice in Canada, Toronto, Butterworths.

HULSMAN, L. et I. BERNAT DE CELIS (1982), Peines perdues: le système pénal en question, Paris, Le Centurion.

KATZENBACK, M. (Président) (1967), The President's Crime Commission on Law Enforcement and Administration of Iustice, Washington, D.C., U.S. Government Printing office.

LANDREVILLE, P. (1983), Normes sociales et normes pénales : notes pour une analyse socio-politique des normes, Les Cahiers de l'École de criminologie, Université de Montréal.

LEBLANC, M., D. SZABO et coll. (1985), la criminologie empirique au Québec, Montréal, P.U.M.

LINDBLOM, C. (1980), The Policy-Making Process, Englewood Cliffs, N.J., Prentice-Hall.

MARTINSON, R. (1974), What works? Questions and Answers about Prison Reform», The Public Interest, p. 22-54. 
MATHIESEN, T. (1974), The Politics of Abolition, London, Martin Robinson.

MORRIS. N. et G. HAWKINS (1970). The Honest Politician's Guide to Crime Control. Chicago. University of Chicago Press.

MORRIS. N. ct G. HAWKINS (1975). A letter to the President of the United States About Crime Control. Chicago, University of Chigaco Press.

NORMANDEAU, A. (1975), "La justice criminelle au Québec : 1975-1980». Crime et/and Justice. p. 75-86.

NORMANDEAU. A. (1976). "Pour une justice sociale-démocrate au Canada, au Québec et ailleurs», Crime et/and /ustice, p. 137-151.

NORMANDEAU, A. (1977), La justice sociale-démocrate au Québec», Crime et/and /ustice. p. 232-237.

NORMANDEAU, A.. I.P. CHARBONNEAU et M. CLAIR (1978), „Pour une vraic politique criminelle», Crime et/and lustice, p. 93-99.

NORMANDEAU, A. (1980), «L'administration de la justice correctionnelle au Québec et au Canada», Crime et/and Justice, p. 131-136.

NORMANDEAU, A. et R. POIRIER (1985), «Pour une gestion alternative des conflits», Revue internationale de criminologie et de police technique (sous presse).

OUIMET, R. (Président) (1969), "Comité canadien de la réforme pénale ct correctionnellen, Justice pénale et correction: un lien à forger. Ottawa, Imprimeur de la reine.

PRÉVOST, Y. (président) (1970), Commission d'enquête sur l'administration de la justice en matière criminelle et pénale au Québec, la Société face au crime, Québec, Éditeur officiel.

RIVERIN, A. et coll. (1981), l'Administrateur public : un être pifométrique, Québec, P.U.Q.

ROCHER, G. (1981), «Le sociologue et la sociologie dans l'administration publique et l'exercice du pouvoir politique», Sociologie et sociétés, p. 45-64.

ROTHMAN, D. (1980), Conscience and convenience: The Asylum and its Alternatives in Progressive America, Boston, Little, Brown.

SHOVER, N. (1979), A Sociology of American Corrections, Homewood, III., The Dorsey Press.

SOLOMON, P. (1981), "The Policy Process in Canadian Justice: A Perspective and Research Agenda», Canadian Journal of Criminology, p. 5-26.

SOLOMON, P. (1983), Criminal /ustice Policy : form Research to Reform, Toronto, Butterworths.

SZABO, D. (1969), Ordre et changement, Montréal, P.U.M.

SZABO, D. (1978), Criminologie et politique criminelle, Paris-Montréal : Vrin-P.U.M.

SZABO, D. (1981), «L'évaluation des politiques criminelles», Revue de science criminelle et de droit pénal comparé, p. 1-25.

SZABO, D. (1984), «Recherches criminologiques, politiques criminelles et les pouvoirs publics au Canada: rétrospective et prospective, 19601985٪, Ottawa, Conseil des Sciences du Canada, 27 p. 
SZABO, D. (1984), "Révolution permanente ou éternel recommencement : la criminologie en situation», in G.H. Lévesque et coll., Continuité et rupture: les sciences sociales au Québec, Montréal : P.U.M., p. 401-434.

TOMASIC, R. et M. FEELEY (édit.) (1982), Neighborhood lustice : Assessment of an Emerging Idea, New York. Longman.

WALKER, S. (1980), Popular Iustice, New York, Oxford University Press.

WALKER, S. (1985), Sense and Nonsense About Crime: A Policy Guide, Monterey, California, Brooks, Cole.

WALLER, I. (1979), "Organizing Research to Improve Criminal Justice Policy : a Perspective from Canadan, Journal of Research in Crime and Delinquency, p. 196-217.

WALLER, I. (1982). "Public Policy on Crime and Criminal Justice: Who Does and Who should Determine It", in F. Elliston et N. Bowie (eds.) Ethics, Public Policy and Criminal Justice, Cambridge, Mass., Oelgeschlager, Gunn and Hain, p. 353-369.

WOODS, G. et H. SIM. (1981), Faits saillants des initiatives fédérales en matière de justice pénale : 1966-1980, Ottawa, Division de la recherche, Solliciteur général du Canada. 